\title{
The effect of activity on stellar temperatures and radii ${ }^{\star}$
}

\author{
J. C. Morales ${ }^{1}$, I. Ribas ${ }^{1,2}$, and C. Jordi ${ }^{1,3}$ \\ 1 Institut d'Estudis Espacials de Catalunya (IEEC), Edif. Nexus, C/Gran Capità, 2-4, 08034 Barcelona, Spain \\ e-mail: morales@ieec.uab.es \\ 2 Institut de Ciències de l'Espai (CSIC), Campus UAB, Facultat de Ciències, Torre C5 - parell - 2a planta, 08193 Bellaterra, Spain \\ e-mail: iribas@ieec.uab.es \\ 3 Departament d'Astronomia i Meteorologia, Universitat de Barcelona, Avda. Diagonal, 647, 08028 Barcelona, Spain \\ e-mail: carme@am.ub.es
}

Received 20 July 2007 / Accepted 13 November 2007

\section{ABSTRACT}

\begin{abstract}
Context. Recent analyses of low-mass eclipsing binary stars have unveiled a significant disagreement between the observations and predictions of stellar structure models. Results show that theoretical models underestimate the radii and overestimate the effective temperatures of low-mass stars but yield luminosities that accord with observations. A hypothesis based upon the effects of stellar activity was put forward to explain the discrepancies.

Aims. In this paper we study the existence of the same trend in single active stars and provide a consistent scenario to explain systematic differences between active and inactive stars in the H-R diagram reported earlier.

Methods. The analysis is done using single field stars of spectral types late-K and $\mathrm{M}$ and computing their bolometric magnitudes and temperatures through infrared colours and spectral indices. The properties of the stars in samples of active and inactive stars are compared statistically to reveal systematic differences.

Results. After accounting for a number of possible bias effects, active stars are shown to be cooler than inactive stars of similar luminosity therefore implying a larger radius as well, in proportions that are in excellent agreement with those found from eclipsing binaries.

Conclusions. The present results generalise the existence of strong radius and temperature dependences on stellar activity to the entire population of low-mass stars, regardless of their membership in close binary systems.
\end{abstract}

Key words. stars: activity - stars: fundamental parameters - stars: late-type - stars: binaries: eclipsing

\section{Introduction}

Structure and evolution of stars in the main sequence is, in principle, well understood. Theoretical models have made very significant progress in the past decades and are able to successfully fulfill most of the observational diagnostics (e.g., Lebreton 2000; Gallart et al. 2005). However, there are still a few open issues that taint the optimistic picture. Most notably, the theoretical modeling of the structure and evolution of stars on both ends of the main sequence is not yet fully resolved (e.g., Ribas 2006a).

Model testing in the low-mass regime has been usually restricted to the evaluation of the mass-luminosity relationship because of the relatively large availability of observational data. Studies such as Delfosse et al. (2000) concluded that models agree well with empirical measurements, especially when the tests are performed in near-infrared bands, which are less affected by metallicity (Chabrier \& Baraffe 2000; Bonfils et al. 2005). The detailed study of the M-type eclipsing binary YY Gem by Torres \& Ribas (2002) contributed to the tests by providing simultaneous and accurate measurements of the component masses, radii, effective temperatures and luminosities. The surprising result was that the components of the system were found to be significantly larger and cooler than the predictions

* Tables 1 and 2 are only available in electronic form at the CDS via anonymous ftp to cdsarc.u-strasbg.fr (130.79.128.5) or via http://cdsweb.u-strasbg.fr/cgi-bin/qcat?]/A+A/478/507 of stellar structure models, but yet with luminosities in agreement with theoretical calculations. The same trend was later confirmed by a number of subsequent analyses of other eclipsing binaries (see Ribas 2006b; and López-Morales 2007, for a complete summary). Thus, a growing body of observational evidence is challenging the adequacy of current stellar models in reproducing the overall properties of low-mass stars.

Using the available data and analysing the possible scenarios, Ribas (2006b) and Torres et al. (2006) concluded that a plausible explanation for the observed discrepancies could be related to stellar activity. In this respect, the active components of eclipsing binaries appear to be larger and cooler than inactive single stars (i.e., those reproduced by theoretical models) while keeping similar luminosity. To first approximation, this means that, regardless of changes in the stellar outer layers, the rate of nuclear burning in the core is not modified by activity and therefore the overall flux is conserved. The mechanism responsible for the observed radius and temperature differences has not been identified yet, although the efficiency of convection in strong magnetic fields (Mullan \& MacDonald 2001), or the simple effect of flux conservation in a spot-covered stellar surface (López-Morales \& Ribas 2005) could explain the observed changes in the stellar properties. Note that a possible correlation of the radius differences with stellar metallicity could also be present (Berger et al. 2006).

The stellar activity hypothesis has been built in the context of close binary systems, where orbital synchronization forces 
the components to rotate fast, therefore triggering high levels of magnetic activity. With a much longer history, the question of the possible differences between active and inactive stars has been a recurrent one (Kuiper 1942; Joy \& Abt 1974). Recent studies have been generally focused on the comparison of radiative properties such as spectral types or photometric colours. The conclusions have been quite diverse, both in favour (Hawley et al. 1996; Amado \& Byrne 1997) and against (West et al. 2004; Bochanski et al. 2005) the existence of systematic colour differences. One of the most conclusive analyses is that of Stauffer \& Hartmann (1986), where the authors identified a separation of the sequences of active and inactive stars in a luminosity-colour plot. The reasons for the observed separation in the sequences could not be unambiguously identified since it could both come from luminosity differences at constant effective temperature, effective temperature differences at constant luminosity, or a combination of the two.

The results from eclipsing binaries provide a new perspective to analyse this long-standing issue since temperatures, radii and luminosities can be determined in a fundamental manner. As discussed above, the components of eclipsing binaries seem to be cooler and larger than model predictions but with luminosities in agreement. Very importantly, this observation is confirmed by the theoretical study of Chabrier et al. (2007), who have recently modified stellar evolution codes to include the effects of stellar activity. These authors also report systematic differences between the properties of active and inactive stars in similar amount and trend as those found from eclipsing binary studies.

With improved statistics with respect to Stauffer \& Hartmann (1986) and the context provided by the new evidence discussed above, we analyse the existence of differences between active and inactive stars of spectral types late-K and $\mathrm{M}$. In the present study we use luminosities directly determined from accurate trigonometric parallaxes and carry out a thorough analysis of possible biases, such as the effect of pre-main sequence (PMS) and binary stars. Also, we interpret the results in physical terms, i.e., effective temperature and radius variations. If active stars were indeed cooler and larger than their inactive counterparts, while keeping similar luminosities, this should be observable in single field stars (in addition to close binaries) thus generalising the proposed stellar activity scenario to all low-mass stars.

\section{Sample of late-K and $M$ stars}

The sample used to test this hypothesis is composed of selected late-K and $M$ dwarfs from the Palomar/Michigan State University survey of nearby stars (hereafter PMSU; Reid et al. 1995; Hawley et al. 1996). This catalog lists the position, $M_{\mathrm{V}}$, distance, $\mathrm{TiO}, \mathrm{CaH}$ and $\mathrm{CaOH}$ spectral indices, $\mathrm{H} \alpha$ equivalent width and proper motions for each of the 1966 stars that it contains. The distances listed are averaged combinations of Hipparcos trigonometric parallaxes and spectrophotometric determinations. Because of our working hypothesis of the $T_{\text {eff }}$ dependence on activity, only objects with direct trigonometric parallaxes are useful to our study because spectroscopic and photometric parallaxes could be biased by activity. The restriction of trigonometric parallaxes reduces the number of stars in the working sample to 746 , with $1.3<d<58.0 \mathrm{pc}$ and $6.65<M_{\mathrm{V}}<16.0 \mathrm{mag}$.

To calculate the bolometric magnitude (i.e., luminosity) of the sample stars we made use of photometry in the $I R$ bands because of the weaker dependence on the bolometric correction $(B C)$, which could be a potential source of large uncertainty (for
M-type stars variations of $200 \mathrm{~K}$ in $T_{\text {eff }}$ produce only changes below $0.1 \mathrm{mag}$ in $B C_{\mathrm{K}}$ ). Thus, the comparison between active and inactive stars with the same luminosity is more reliable if $M_{\text {bol }}$ is computed from the $K$ band rather than the $V$ band. The former is also less affected by variability caused by surface inhomogeneities. The sample was cross-matched with the 2MASS and ROSAT survey catalogs to obtain $J, H$ and $K_{\mathrm{s}}$ magnitudes and $L_{\mathrm{X}}$ for each star. $K_{\mathrm{s}}$ was transformed to the $K$ Johnson band (Alonso et al. 1994) to compute $B C_{\mathrm{K}}$ using the models in Bessell et al. (1998) as a function of $T_{\mathrm{eff}}$. The available trigonometric distances were used to compute $M_{\mathrm{K}}$ and, subsequently, $M_{\mathrm{bol}}$. The TiO5 index was used as spectral type indicator with the prescription on Reid et al. (1995), which suggests a linear relationship with small scatter between this spectral index and the spectral subtype of M stars (Sp.Typ. $=-10.775 \cdot$ TiO5 + 8.2). Effective temperatures were derived using the spectral type-temperature correspondence in Bessell (1991) - which is similar to that of Leggett et al. (1996) - and the equivalent width of the $\mathrm{H} \alpha$ emission line was used as an indicator of magnetic activity. An iterative procedure was used to ensure consistency between the adopted $T_{\text {eff }}$ and $B C_{\mathrm{K}}$.

\section{Discussion}

Active and inactive stars were considered separately in the analysis of the sample. Those stars with the $\mathrm{H} \alpha$ line in absorption were classified as inactive while those with $\mathrm{H} \alpha$ in emission were considered to be active. Note that this criterion only identifies as active stars those with high levels of activity, since mildly active stars can still have $\mathrm{H} \alpha$ in absorption (e.g., Cram \& Mullan 1979). Following this approach, 72 stars out of the total 746 turn out to be active. In Tables 1 and 2, available electronically from CDS, we provide the lists of active and inactive stars, respectively, together with the parameters relevant to the present study.

It is important to emphasise that our analysis is only meaningful if both sets of active and inactive single stars are equivalent in terms of evolution and metallicity. In the case of late$\mathrm{K}$ and M-type stars this means that the calculations need to be restricted to main sequence stars. The relatively short PMS evolutionary phase, in which stars are also magnetically active, would break the one-to-one correspondence between luminosity and mass (see below) and therefore invalidate the comparisons. Thus, we cleaned the active star sample from possible PMS objects. There are only a few known young star associations and moving groups in the solar neighbourhood and these have welldefined space motions. Using the catalogs of young moving groups (López-Santiago et al. 2006; Fernández, priv. comm.), we removed a total of 22 stars ( 9 of them classified as active) from the sample that belong to ensembles with ages younger than 200 Myr. Since this is a crude approach, we carried out an independent theoretical estimation of the expected number of PMS stars as a function of mass for comparison. Using the models of Baraffe et al. (1998) and assuming a constant star formation rate in the solar neighbourhood, we estimated the fraction of PMS stars just by calculating the ratio of the time spent in the PMS phase and the total time during which we would classify the star as active. This calculation yielded a fraction of PMS stars of $20 \%$ to $10 \%$ (decreasing with mass) for our sample parameters. Such values turn out to be close to the actual fractions found when considering kinematic restrictions. For similar reasons, low-metallicity halo stars could also alter the results of the inactive star bins. We removed high-velocity stars and also subdwarfs using the $\mathrm{CaH} 2-\mathrm{TiO} 5$ prescription given in Bochanski et al. (2005). A total of 7 stars were eliminated at this step. In 


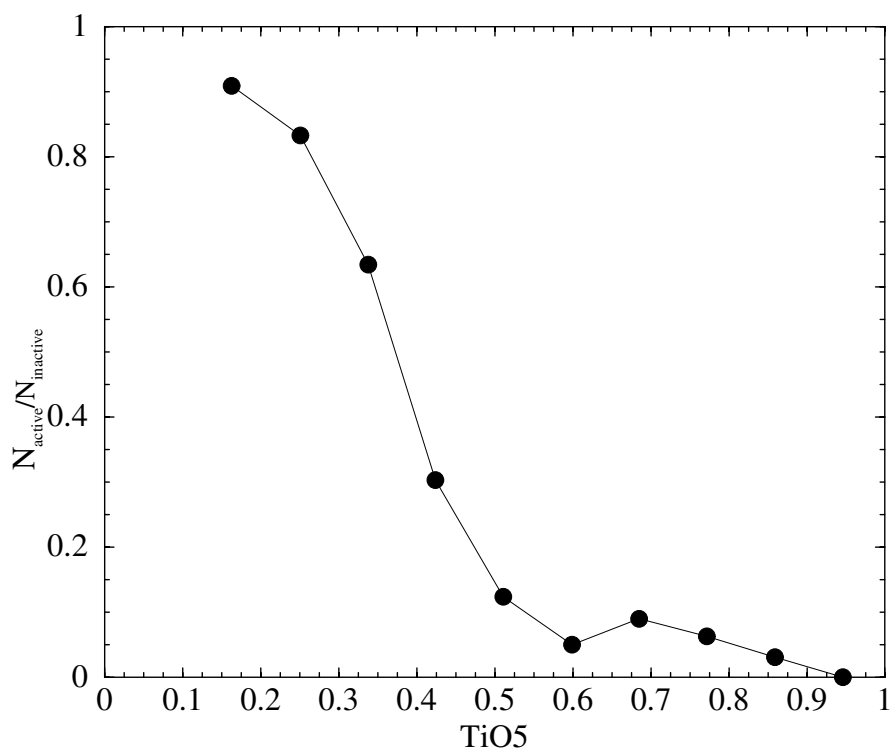

Fig. 1. Ratio of active to inactive stars as function of the TiO5 index for the full PMSU sample. Active stars are defined as those with $\mathrm{H} \alpha$ emission.

this way, both the inactive and active star samples contain disk stars and should have largely similar metallicities. Finally, we removed close binary stars from the list since we aimed to test if single active stars show the same discrepancies with the models as close binaries. We identified and rejected a total of 22 binaries (14 with $\mathrm{H} \alpha$ in emission) using SIMBAD and the lists given in Gizis et al. (2002) for the PMSU survey. The last columns of Tables 1 and 2 include detailed information on the stars that have been rejected using these criteria.

We grouped the stars in $M_{\text {bol }}$ (i.e., luminosity) bins. The bin size was selected to be 1 magnitude to keep a statistically significant number of stars in each bin. Note that, since the massluminosity relationship is so tight for low-mass stars (because post-ZAMS evolution is so slow), mass and luminosity bins are equivalent. Because of the need to use fairly large $M_{\text {bol }}$ bins, our results could be potentially affected by some biases and selection effects. Firstly, a selection effect could be caused by the use of $\mathrm{H} \alpha$ emission as activity criterion, which is more sensitive for stars of lower photospheric luminosities. Secondly, it is well known that the number of active stars increases towards later spectral types, i.e., decreasing effective temperature (e.g., West et al. 2004), which could tend to give more weight to the cooler active stars in each luminosity bin. Fortunately, both effects can be corrected using the statistics available in the PMSU sample itself. We have calculated the ratio of active to inactive stars as a function of the TiO5 index, as shown in Fig. 1, in which active stars are selected using the $\mathrm{H} \alpha$ emission criterion. To correct for the biases, the parameters related to active stars are weighted with the quantity $N_{\text {inactive }} / N_{\text {active }}$ estimated at their respective TiO5 index values in all subsequent calculations. The correction has a small - albeit non negligible - effect on the mean TiO5 indexes of the bins. As a check, we have calculated the same corrections using the active star ratios in West et al. (2004) and Bochanski et al. (2005) and the results are identical.

Figure 2 illustrates the distribution of stars in the TiO5 versus $\mathrm{H} \alpha$ equivalent width $(E W)$ diagram $(\mathrm{H} \alpha E W=0$ means line in absorption) for the 695 single main sequence, disk stars (48 active and 647 inactive). $M_{\text {bol }}$ bins with few stars (typically 2 or less) were not considered in the figure and subsequent
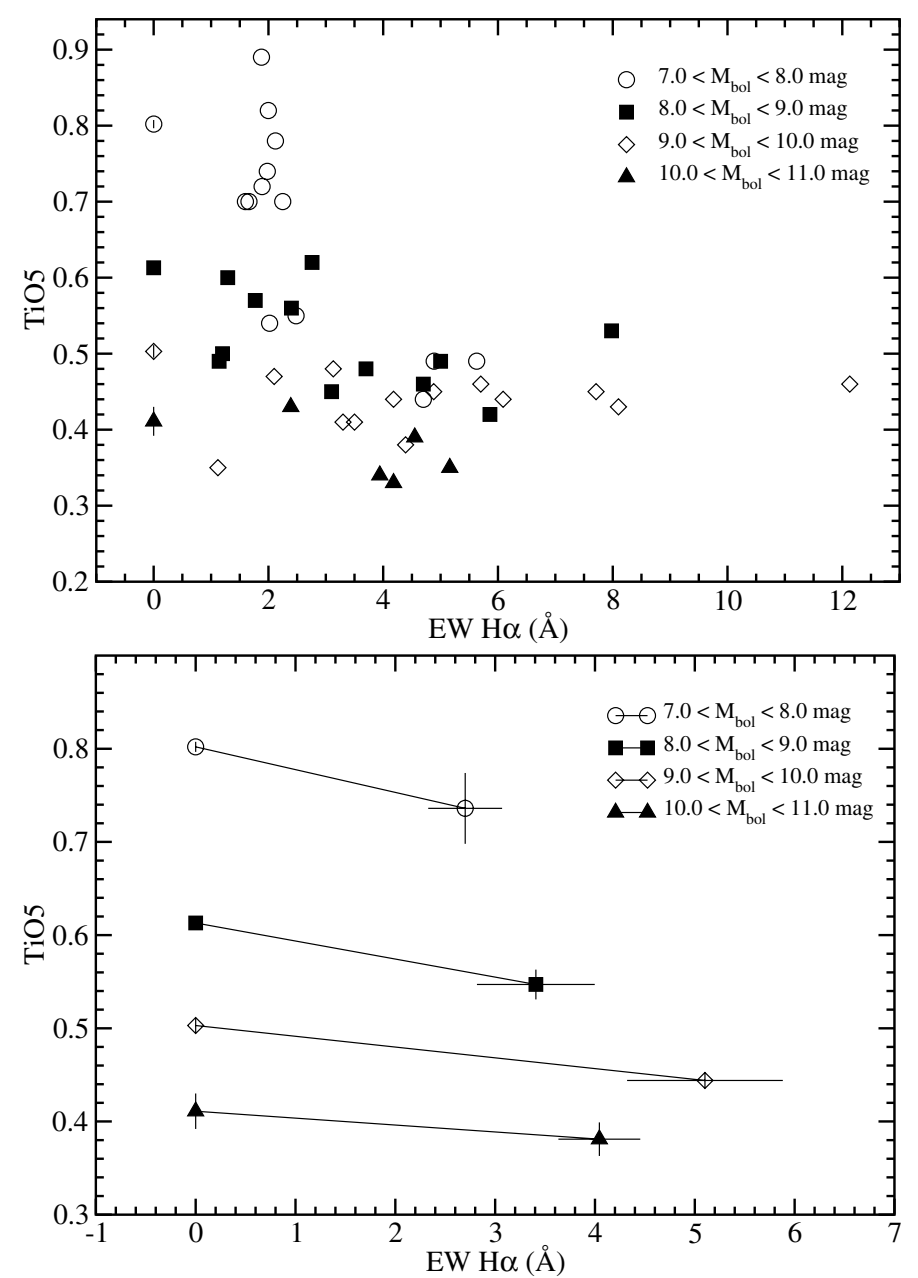

Fig. 2. Distribution of active and inactive stars in TiO5 vs. $E W \mathrm{H} \alpha$ for bins with statistical significance. Top: single values for each active star and mean values for inactive stars. Bottom: mean values for each $M_{\mathrm{bol}}$ bin.

comparisons and have not been reported in Table 3. The average TiO5 index for each $M_{\text {bol }}$ bin is shown in Fig. 2 for active and inactive stars separately. In Table 3 the differences between active and inactive stars are listed for statistically significant bins. Also provided are the average values of $\log \left(L_{\mathrm{X}} / L_{\mathrm{bol}}\right)$ for active stars, which are at the saturation level (e.g., Pizzolato et al. 2003) for all luminosity bins. This indicates that our active sample is representative of stars with very high activity levels. It is obvious both from the plot and from the table that active stars have systematically lower TiO5 indices (i.e., lower effective temperatures) than their inactive counterparts of similar luminosity.

The mean TiO5 differences in Table 3 are all positive and quite similar in all magnitude bins. These index differences can be transformed into temperature differences using the calibrations described above and eventually into radius differences just by assuming a fixed luminosity $\left(L_{\text {active }} \simeq L_{\text {inactive }}\right)$ :

$\frac{R_{\mathrm{ac}}-R_{\mathrm{inac}}}{R_{\mathrm{inac}}}=\left(\frac{T_{\mathrm{eff}, \text { inac }}}{T_{\mathrm{eff}, \mathrm{ac}}}\right)^{2}-1$.

These values are also listed in Table 3.

Since differences in radius are computed through temperature ratios and we are carrying out a differential study, the results are almost independent of the $T_{\text {eff }}$ scale adopted, which is still controversial for M-type stars. A cross-check 
Table 3. Differences between mean values for active and inactive stars for each bin of $M_{\mathrm{bol}}$ with statistical significance.

\begin{tabular}{cccccccc}
\hline \hline$M_{\text {bol }}$ bin & $N_{\text {inactive }}$ & $N_{\text {active }}$ & $\langle\Delta$ TiO5 $\rangle$ & $\left\langle\Delta T_{\text {eff }}\right\rangle(\mathrm{K})$ & $\langle\Delta R / R\rangle(\%)$ & $\langle\Delta(V-K)\rangle$ & $\left\langle\log \left(L_{X} / L_{\text {bol }}\right)\right\rangle_{\text {active }}$ \\
\hline $7.0-8.0$ & 286 & 13 & $-0.066 \pm 0.038$ & $-128 \pm 62$ & $6.9 \pm 3.5$ & $0.34 \pm 0.26$ & $-3.11 \pm 0.03$ \\
$8.0-9.0$ & 208 & 12 & $-0.066 \pm 0.018$ & $-107 \pm 29$ & $6.3 \pm 1.8$ & $0.31 \pm 0.07$ & $-3.19 \pm 0.11$ \\
$9.0-10.0$ & 72 & 13 & $-0.059 \pm 0.012$ & $-118 \pm 22$ & $7.3 \pm 1.4$ & $0.31 \pm 0.08$ & $-2.87 \pm 0.14$ \\
$10.0-11.0$ & 13 & 5 & $-0.030 \pm 0.026$ & $-59 \pm 50$ & $3.8 \pm 3.3$ & $0.30 \pm 0.24$ & $-3.30 \pm 0.11$ \\
\hline
\end{tabular}

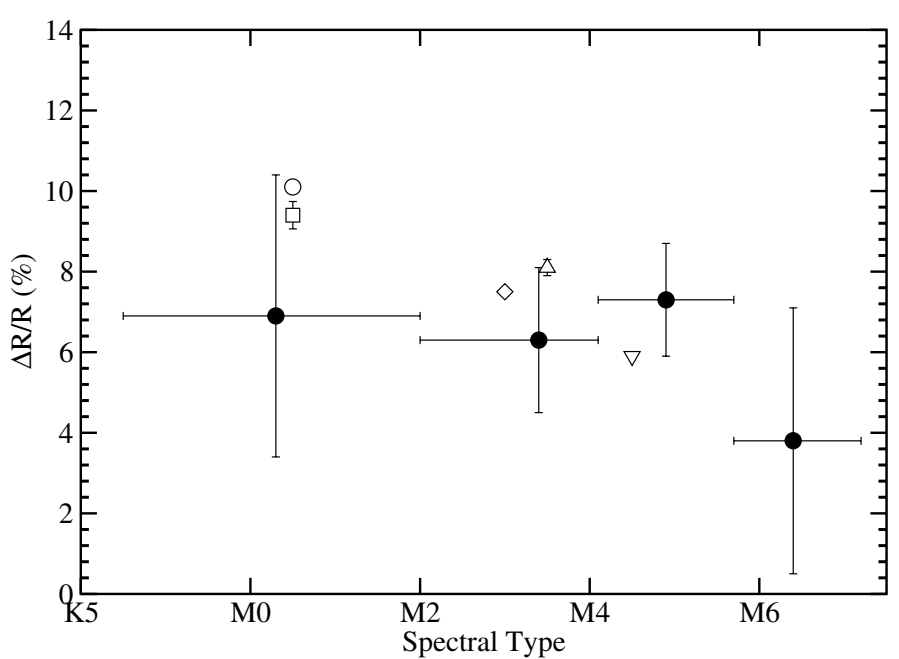

Fig. 3. Comparison of the differentials of radii over spectral type (filled circles) with results from the eclipsing binaries with highest accuracy (open symbols): YY Gem (circle), GU Boo (square), NSVS01031772 (diamond), CU Cnc (up triangle) and CM Dra (down triangle).

with the $T_{\text {eff }}$ calibrations of Schmidt-Kaler (1982) and de Jager $\&$ Nieuwenhuijzen (1987) expectedly yielded the same results. The trend of lower temperatures and larger radii for active stars has high statistical significance since similar differentials are obtained in all luminosity bins. In Fig. 3 we show the calculated radius differences translated into spectral type bins and together with the radius differences obtained from a sample of eclipsing binaries with data of best quality (taken from López-Morales et al. 2006; and López-Morales 2007). Note that the two radius differentials have slightly different meanings. While eclipsing binary values come from the direct comparison of radius measurements with the predictions of theoretical models of Baraffe et al. (1998) - which do not include the effects of stellar activity, - the values for single stars are computed from the difference between active and inactive samples. As can be seen, the differentials from these two completely independent approaches are in very good agreement.

To rule out a possible effect of the $M_{\text {bol }}$ binning procedure itself on the differences reported, we used an alternative approach using a polynomial fit. We first calculated a bestfitting third order polynomial of the form $T_{\text {eff }}=f\left(M_{\text {bol }}\right)$ for the inactive star sample. Then, for each individual active star we calculated the temperature difference (and radius difference) between the observed value and the one predicted by the polynomial fit. A graphical representation of the results is provided in Fig. 4, where the individual temperatures and radius differences are shown together with a running average of 10 points and averages computed for each $M_{\mathrm{bol}}$ bin. The actual values are given in Table 4, and are very similar to those in Table 3, which were calculated from $M_{\text {bol }}$ bins. An interesting feature of Fig. 4 is the relatively large scatter at $M_{\text {bol }}<8.5 \mathrm{mag}$, which occurs near the
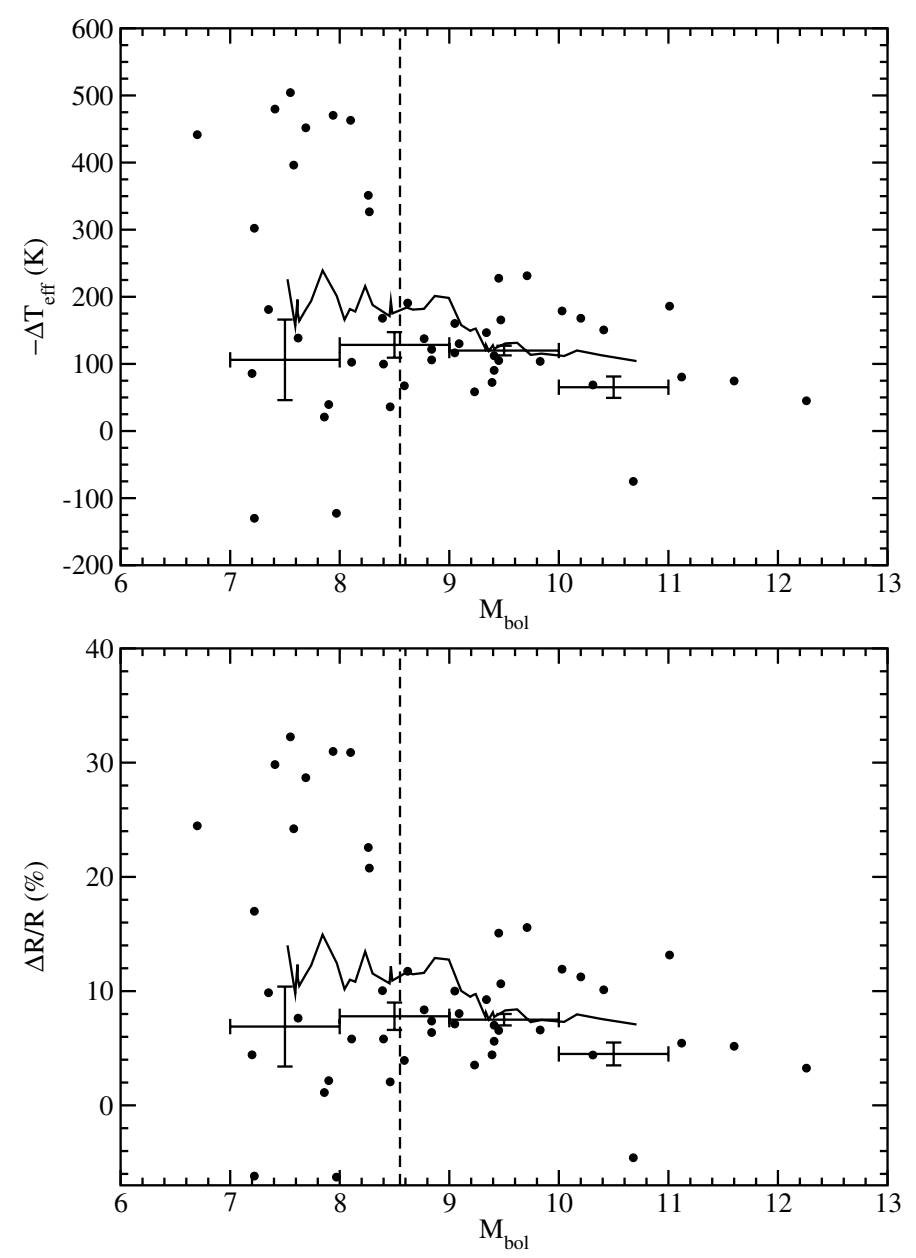

Fig. 4. Differentials of $T_{\text {eff }}$ (top) and $R$ (bottom) vs. $M_{\text {bol }}$ computed from a polynomial fit to the $T_{\text {eff }}$ of inactive stars. Solid lines are 10-point running averages and the error bars show the averages for four relevant $M_{\text {bol }}$ intervals. The dashed line marks the limit between fully convective stars and those with a radiative core.

Table 4. Effective temperature and radius differences of active stars calculated from a polynomial fit to inactive stars.

\begin{tabular}{cccc}
\hline \hline$M_{\text {bol }}$ bin & $N_{\text {active }}$ & $\left\langle\Delta T_{\text {eff }}\right\rangle(\mathrm{K})$ & $\langle\Delta R / R\rangle(\%)$ \\
\hline $7.0-8.0$ & 13 & $-106 \pm 60$ & $6.9 \pm 3.5$ \\
$8.0-9.0$ & 12 & $-128 \pm 19$ & $7.8 \pm 1.2$ \\
$9.0-10.0$ & 13 & $-120 \pm 7$ & $7.5 \pm 0.5$ \\
$10.0-11.0$ & 5 & $-65 \pm 16$ & $4.5 \pm 1.0$ \\
\hline
\end{tabular}

value where models predict the change between fully convective stars and those with a radiative core.

A further useful check of the results comes from restricting the analysis to only stars that have been explicitly classified as single through high-resolution spectroscopy (Gizis et al. 2002). Although the statistics are less significant (with 127 inactive and 
19 active stars), the mean temperature and radius differences for each $M_{\text {bol }}$ bin are within one sigma of those in Table 3.

In Table 3 we also include the mean differences in the $(V-K)$ colour index, which are a direct consequence of the different average $T_{\text {eff }}$ values between active and inactive stars. The relatively large scatter of these means (especially that of the first $M_{\text {bol }}$ bin) may be caused by the variability in the $V$ band of the stars in the active sample. This stems from the existence of surface spots with various cycles that could affect single-epoch $V$-band measurements but not $K$-band measurements that are more immune to spot-induced variability. Note that no obvious or only marginal colour differences for active stars have been reported before (see, e.g., Hawley et al. 1996; Bochanski et al. 2007). This would seem to stand in contradiction with our results, but it is not. It is important to emphasise that such colour comparisons are always carried out using spectral types as fiducials. Thus, the lack of colour differences between active and inactive stars of the same spectral types is just revealing that they have the same temperatures and spectral energy distributions, which is not surprising. The colour differences we find correspond to active and inactive stars of the same luminosity, which is equivalent to mass, and therefore a fundamental stellar property. Further, these are in good agreement with the differences in the spectral energy distribution caused by activity found by Stauffer et al. (2003) in Pleiades K-type stars.

A possible effect that could alter the outcome of this study is the influence of metallicity. Obviously, a metal-poor star will have intrinsically weaker $\mathrm{TiO}$ bands and therefore appear as hotter when the TiO5 index is used. For example, a metallicity decrease of 0.5 dex corresponds to a $\sim 200 \mathrm{~K}$ higher effective temperature. If there was a mean metallicity difference between the two samples used (active and inactive) this could affect their mean temperature difference (and inferred radius difference) based on the TiO5 index. There is certainly a metallicity spread within the two samples used, characteristic of a disk population, but the key issue here is whether the two samples have similar mean metallicities. A possible approach to test this is by directly using the metallicity calibration of Bonfils et al. (2005). However, this calibration is based in the $V-K$ index and, according to our hypothesis of an activity effect, the systematic differences in this index will cause a spurious systematic metallicity difference.

It is reasonable to assume that, on average, the inactive sample will be older than the active sample, just because there is a well-established age-activity relationship (e.g., Skumanich 1972). However, this age difference does not imply a difference in the mean metallicities of both samples because numerous studies have concluded that there is no age-metallicity correlation in disk stars, as shown by Nordström et al. (2004) and references therein. The average metallicity of the Nordström et al. catalog, when applying the kinematic constraints of our sample (i.e., $-165<U<130 \mathrm{~km} \mathrm{~s}^{-1},-130<V<40 \mathrm{~km} \mathrm{~s}^{-1}$ and $-90<W<80 \mathrm{~km} \mathrm{~s}^{-1}$ ), is $[M / H]=-0.17$. If we use the Bonfils et al. (2005) calibration in our inactive sample, whose stars should have unbiased $(V-K)$ indices, we obtain $[\mathrm{Fe} / \mathrm{H}]=-0.14$. Both averages are in good agreement and indicate that our inactive sample is representative of the overall population of the solar neighbourhood. If we apply the Bonfils et al. calibration to the active sample, also under the assumption that their $(V-K)$ indices are unbiased, we find a mean metallicity that is about 0.3 dex higher (i.e., $[\mathrm{Fe} / \mathrm{H}] \sim+0.15$ ). Such high mean metallicity value is very unlikely in the context of the Nordström et al. results, thus suggesting that the $(V-K)$ indices of the active star sample are indeed biased because of the effect of activity.
Table 5. Active/inactive star radius differences using restrictive kinematic criteria (see text for further details).

\begin{tabular}{ccc}
\hline \hline$M_{\text {bol }}$ bin & $N_{\text {inactive }} / N_{\text {active }}$ & $\langle\Delta R / R\rangle(\%)$ \\
\hline $7.0-8.0$ & $68 / 8$ & $11.6 \pm 4.2$ \\
$8.0-9.0$ & $39 / 3$ & $9.1 \pm 2.9$ \\
$9.0-10.0$ & $19 / 5$ & $4.9 \pm 2.3$ \\
$10.0-11.0$ & $1 / 3^{1}$ & \\
\hline
\end{tabular}

${ }^{1}$ Mean not computed because of insufficient statistics.

We carried out additional tests to eliminate the possibility of metallicity effects in our results arising from contamination by metal-poor stars belonging to the thin disk. When selecting only stars with $U V$ values in the interval $(-90,50) \mathrm{km} \mathrm{s}^{-1}$, which would be characteristic of the overall thin disk, the results are nearly identical to those in Table 3 . We even considered a further test by sacrificing statistical significance but adopting very restrictive kinematic criteria in both the active and inactive star samples to ensure that both belong to the young disk population. If metallicity was responsible for the observed differences in the radii and temperature, we should expect them to disappear when both samples come from the same population. Thus, we selected stars belonging to the young disk using the $U V$ criteria of Montes et al. (2001) plus $-25<W<25 \mathrm{~km} \mathrm{~s}^{-1}$. The resulting radius differences are listed in Table 5 . As can be seen, the differences are fully compatible within the error bars with those of full sample, therefore indicating that metallicity effects do not play a significant role in our conclusions.

Stars with direct radius measurements will provide the ultimate proof of the systematic radius deviations with activity. The first steps in this direction have already been taken by López-Morales (2007), who used eclipsing binaries together with stars whose radius has been measured by interferometry. Unfortunately, data are still scarce to draw any conclusions. This is specially true in the case of stars with interferometric radii since they all have very low activity levels and thus provide no baseline to extract any relevant trends.

An interesting consequence of the impact of activity on effective temperatures and radii is a possible bias in the determination of stellar ages from evolutionary models. This is especially relevant in the context of young open clusters. As discussed by Torres \& Ribas (2002) for the M-type eclipsing binary YY Gem, the age of active stars could appear systematically younger if a comparison with models was made using effective temperatures and radii. Such systematic effect could be as large as a factor of 2 for early M-type stars. However, the ages of young clusters are commonly determined through analysis of a colour-magnitude diagram or, conversely, using luminosity and effective temperature. Results indicate that the luminosities of active stars are not biased with respect to those of their inactive counterparts, but the effective temperatures are. The CMD diagram would thus appear shifted to cooler temperatures, an effect that is beautifully illustrated by the comparison of the Pleiades and Praesepe in the $M_{v}$ vs. $(V-K)$ diagram (Stauffer et al. 2003). Young clusters analysed only in the region of the CMD where activity plays a role (i.e., G-, K-, and M-type stars) might yield ages compatible with a PMS phase while they are indeed already past the zeroage main sequence. This should not affect young cluster ages determined from the position of the upper main sequence turn-off since this occurs at spectral types where stars have a radiative envelope and are therefore magnetically inactive. However, it may have an influence on studies using isochrones in the CMD of the 
lower main sequence (e.g., Jeffries et al. 2005). A simple calculation using our temperature differentials indicates that ages of young clusters determined from (active) low-mass stars could be systematically underestimated by about $40 \%$. Interestingly, this systematic effect agrees well with the discrepancy between CMD ages and Li depletion boundary ages in young clusters (e.g., Barrado y Navascués et al. 2004).

\section{Conclusions}

The results of the analysis fully agree with the findings from low-mass eclipsing binaries. Therefore, the radius and temperature discrepancies found in the latter are not a consequence of their mutual interactions as being in close binaries but can be generalised to the entire population of low-mass stars. By this scenario, any active star (either single or binary) would be equally affected by the mechanism responsible for lowering its photospheric temperature and increasing its radius, while keeping its luminosity roughly constant. In this work we thus provide solid ground for the interpretation of the differences between active and inactive stars pointed out already by Kuiper (1942), and later studied in more detail by Stauffer \& Hartmann (1986). The observed separation between the active and inactive star sequences in the H-R diagram is caused by systematic temperature (or spectral type) rather than luminosity deviations. Our conclusions stem from a comprehensive analysis using a large sample of stars with accurate trigonometric parallaxes, and therefore the results are statistically relevant.

In a recent study, Torres (2007) has determined the mass and radius of the M2.5 transiting planet host GJ 436 from the stellar radiative properties and constraints from the transit. The resulting radius also seems to be larger than model predictions by about $10 \%$ yet the star does not show any strong activity. Note that the masses and radii determined in this way are not fundamental (as opposed to those coming from eclipsing binaries) and that they heavily rely on the assumed value of the effective temperature, which is not well established for M-type stars as discussed above. Thus, the comparison with stellar models may not be as meaningful as that resulting from classical eclipsing binaries. However, with the expected increase in the number of $M$ stars with transiting planets, this method can provide valuable data to further evaluate the activity effects.

If we generalise the activity-related differences found in single and binary stars we conclude that current stellar structure and evolution models are not adequate to describe the physical (radii) and radiative (effective temperature, colour indices) properties of all active low-mass stars. The problem is particularly severe for the analysis of open clusters or star forming regions, where low-mass stars may display high-levels of magnetic activity. This is because there exists a good correlation between activity and age, in the sense that younger stars rotate faster than their older counterparts (e.g., Hartmann et al. 1984) and thus have more efficient mechanisms to generate magnetic energy. In the particular case of M-type stars (i.e., masses below $\sim 0.6 M_{\odot}$ ), which stay active up to relatively long rotation periods (Pizzolato et al. 2003) and, conversely, old ages (up to $1 \mathrm{Gyr}$ or more), the discrepancy between models and observations may apply to the entire young disk population. We are currently working on a realistic implementation of the effects of magnetic activity in theoretical structure and evolution models to resolve the observed shortcomings. The appropriate framework in this direction is being established by Chabrier et al. (2007).

Acknowledgements. We are grateful to John Bochanski for providing us with valuable input to carry out this work. The referee, John Stauffer, is gratefully acknowledged for extremely useful and constructive suggestions. The authors acknowledge support from the Spanish Ministerio de Educación y Ciencia via grants AYA2006-15623-C02-01 and AYA2006-15623-C02-02. This publication makes use of data products from the Two Micron All Sky Survey, which is a joint project of the University of Massachusetts and the Infrared Processing and Analysis Center/California Institute of Technology, funded by the National Aeronautics and Space Administration and the National Science Foundation.

\section{References}

Alonso, A., Arribas, S., \& Martínez-Roger, C. 1994, A\&AS, 107, 365 Amado, P. J., \& Byrne, P. B. 1997, A\&A, 319, 967

Baraffe, I., Chabrier, G., Allard, F., \& Hauschildt, P. H. 1998, A\&A, 337, 403 Barrado y Navascués, D., Stauffer, J. R., \& Jayawardhana, R. 2004, ApJ, 614, 386

Berger, D. H., Gies, D. R., McAlister, H. A., et al. 2006, ApJ, 644, 475

Bessell, M. S. 1991, AJ, 101, 662

Bessell, M. S., Castelli, F., \& Plez, B. 1998, A\&A, 333, 231

Bochanski, J. J., Hawley, S. L., Reid, I. N., et al. 2005, AJ, 130, 1871

Bochanski, J. J., West, A. A., Hawley, S. L., \& Covey, K. R. 2007, AJ, 133, 531

Bonfils, X., Delfosse, X., Udry, S., et al. 2005, A\&A, 442, 635

Chabrier, G., \& Baraffe, I. 2000, ARA\&A, 38, 337

Chabrier, G., Gallardo, J., \& Baraffe, I. 2007, A\&A, 472, 17

Cram, L. E., \& Mullan, D. J. 1979, ApJ, 234, 579

de Jager, C., \& Nieuwenhuijzen, H. 1987, A\&A, 177, 217

Delfosse, X., Forveille, T., Ségransan, D., et al. 2000, A\&A, 364, 217

Gallart, C., Zoccali, M., \& Aparicio, A. 2005, ARA\&A, 43, 387

Gizis, J. E., Reid, I. N., \& Hawley, S. L. 2002, AJ, 123, 3356

Hartmann, L., Soderblom, D. R., Noyes, R. W., Burnham, N., \& Vaughan, A. H. 1984, ApJ, 276, 254

Hawley, S. L., Gizis, J. E., \& Reid, I. N. 1996, AJ, 112, 2799

Jeffries, R. D., \& Oliveira, J. M. 2005, MNRAS, 358, 13

Joy, A. H., \& Abt, H. A. 1974, ApJS, 28, 1

Kuiper, G. P. 1942, ApJ, 95, 201

Lebreton, Y. 2000, ARA\&A, 38, 35

Leggett, S. K., Allard, F., Berriman, G., Dahn, C. C., \& Hauschildt, P. H. 1996, ApJS, 104, 117

López-Morales, M. 2007, ApJ, 660, 732

López-Morales, M., \& Ribas, I. 2005, ApJ, 631, 1120

López-Morales, M., Orosz, J. A., Shaw, J. S., et al. 2006, ApJ, submitted [arXiv: astro-ph/0610225]

López-Santiago, J., Montes, D., Crespo-Chacón, I., \& Fernández-Figueroa, M. J. 2006, ApJ, 643, 1160

Montes, D., López-Santiago, J., Gálvez, M. C., et al. 2001, MNRAS, 328, 45

Mullan, D. J., \& MacDonald, J. 2001, ApJ, 559, 353

Nordström, Mayor, M., Andersen, J., et al. 2004, A\&A, 418, 989

Pizzolato, N., Maggio, A., Micela, G., Sciortino, S., \& Ventura, P. 2003, A\&A, 397,147

Reid, I. N., Hawley, S. L., \& Gizis, J. E. 1995, AJ, 110, 1838

Ribas, I. 2006a, in Astrophysics of Variable Stars, ed. C. Sterken, \& C. Aerts (San Francisco: ASP), ASP Conf. Ser., 349, 55

Ribas, I. 2006b, Ap\&SS, 304, 89

Schmidt-Kaler, T. 1982. in Landolt-Börnstein, ed. K. Schaifers, \& H. H. Voight, Vol. 2b (Springer, Heidelberg), 14

Skumanich, A. 1972, ApJ, 171, 565

Stauffer, J. R., \& Hartmann, L. W. 1986, ApJS, 61, 531

Stauffer, J. R., Jones, B. F., Backman, D., et al. 2003, AJ, 126, 833

Torres, G. 2007, ApJ, in press [arXiv: 0710.4883]

Torres, G., \& Ribas, I. 2002, ApJ, 567, 1140

Torres, G., Lacy, C. H., Marschall, L. A., Sheets, H. A., \& Mader, J. A. 2006, ApJ, 640, 1018

West, A. A., Hawley, S. L., Walkowicz, L. M. et al. 2004, AJ, 128, 426 